\title{
Processes of verbal memorization revealed in correct and erroneous recall of words and paralogs'
}

\author{
Burton G. Andreas and Elizabeth Deutsch \\ UNIVERSITY OF ROCHESTER
}

\begin{abstract}
Abstraet
Paralog $(\mathrm{C}-\mathrm{V}-\mathrm{C}-\mathrm{V}-\mathrm{C})$ distortions of real words were memorized in mixed lists no better than randomly generated paralogs. Reversed words apparently were unrecognized by most Ss. Error patterns permit inferences concerning memory encoding processes.

\section{Problem}

Although it is established that meaningful words are more readily memorized than nonsense syllables or paralogs, an intermediate case in which paralogs are created by distortions of real words needs to be studied. If the distortions were detected by Ss, then transformation back to real words might occur as a useful aid to memory storage. Errors may be studied as a basis for inferring techniques of memorization and retention used. The utility of error study has been repeatedly demonstrated, e.g., by Jensen (1962) who found a serial position effect in spelling errors, by Rozov (1959) who noted lawful verbal processes in recall and recognition errors, and by Underwood \& Hughes (1950) who found generalization gradients in recall errors based on semantic similarity.
\end{abstract}

\section{Method}

Ss were 35 female students from introductory psychology. They were run as a group, being asked to memorize a mixed list of 32 verbal items, each of the form $\mathrm{C}-\mathrm{V}-\mathrm{C}-\mathrm{V}-\mathrm{C}$. The list consisted of 8 real words, 8 reversed-word paralogs, 8 paralogs created by randomly changing one letter of a real word, and 8 randomly generated paralogs. A constraint on randomness was the non-use of $j, k, q, x$ and $z$.

With two items of each type randomly placed in each of four columns, the list was projected for the Study stage of each of the five acquisition trials. Each trial consisted of four stages: Study, 3 min.; Retention, 1 min.; Written Recall, 3 min.; Rest, 3 min. During Retention, Ss sat quietly until instructed to open their folders for Recall during which they printed the items they could recall, incidentally continuing to study these. Folders were closed after $3 \mathrm{~min}$. and were exchanged for blank ones during Rest, which functioned as the conventional intertrial interval.

In anticipation of error analysis, E encouraged Ss to strive for recall even where unsure of an item in its entirety: "You will receive partial credit for items that are partly correct, so if you recall an item with some uncertainty, print the part which you know and complete the item with your best possible guess as to its spelling. Full credit will be given for those items which are correctly spelled."
Ss were asked to return the next day for "another experiment in the series." They were actually given a 24-hr. unaided recall test, with partial credit again being offered for partial successes.

\section{Results}

As seen in Fig. 1, the meaningful words were more readily memorized and retained than were the three types of paralogs. However, the paralogs derived from real words by either reversal or one-letter change were no more readily acquired and remembered than were the paralogs generated randomly. The statistical significance $(p<.05)$ of the superiority of real-word learning and of the lack of difference among the paralog types was established by analysis of variance.

Post-experimental inquiry revealed that only 8 of the 35 Ss had noticed that some of the paralogs were real words reversed. These Ss who noticed this showed some superiority in learning the 8 reversed-word paralogs. On Trials 2 and 3, they averaged 1.4 and 1.5 more of these items correct, respectively, than the $27 \mathrm{Ss}$ who

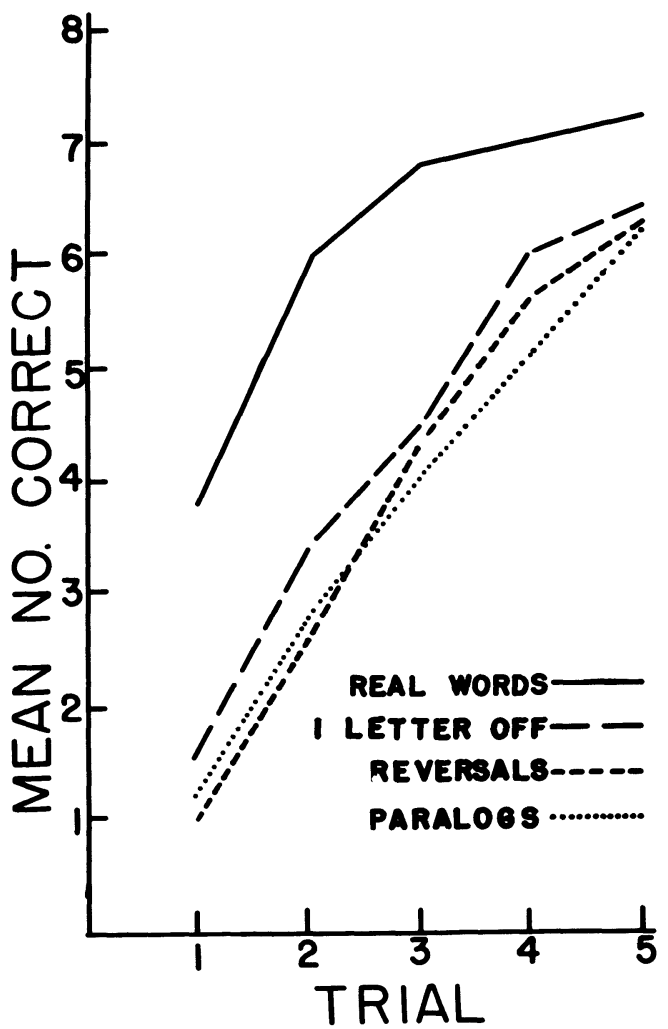

Fig. 1. Acquisition curves for real words and three types of nonsense paralogs. $(\mathrm{N}=35)$ 
Number of Ss Making Each Type of Error, Compiled by Locus within Paralog, Over All Trials plus Retention Test

\author{
Type of Error \\ a. One letter only \\ b. Vowel transposition \\ c. Consonant transposition \\ d. Both vowe1s wrong \\ e. Two consonants wrong \\ f. Vowels transposed, wrong consonant \\ g. Prefix wrong \\ h. 2-letter suffix \\ i. 3-letter suffix \\ j. One vowel, one consonant
}

Total per locus:

did not report noticing this fact. On Trials 1 and 4, and on recall, their margin of superiority was less and on Trial 5, the non-noticing group did slightly better on these 8 items. Statistical significance for the general superiority on these items of the noticing group was demonstrated in an analysis of variance. A $t$ test indicated significance of difference on Trial $2(t=2.04$; $\mathrm{df}=33 ; \mathrm{p}<.05)$, while differences on other acquisition trials and on recall were not significant. The group noticing reversed-word paralogs did not exceed the nonnoticing groups when performance over all 32 items was assessed.

Post-experimental inquiry showed that 16 of the $35 \mathrm{Ss}$ had noticed that some of the paralogs were real words except for a one-letter change. However, they did no better at the memory task, either on this type of item or over all items, than the $19 \mathrm{Ss}$ who did not report noticing this type of item.

Erroneous written recall of paralogs, encouraged by instructions to strive for partial credit, occurred with a frequency great enough to permit some inferences about techniques of encoding for purposes of retention. The specific errors made at some point by $15 \%$ or more of Ss were as follows: COMEN instead of COMEM (N = $8 \mathrm{Ss})$, More common terminal letter, perhaps induced by easier covert vocalization; YAWUT instead of YOWUT $(\mathrm{N}=7 \mathrm{Ss})$, Ambiguity of covert vocalization; YACES instead of YACED ( $N=6 \mathrm{Ss})$, Change in ending of the nonsense verb YACE; WAGIN instead of WIGON $(\mathrm{N}=6$ $\mathrm{Ss})$, One letter different from real word, wagon; SOBER instead of SOBOR ( $N=6 \mathrm{Ss})$, Loss in encoding after item was noted to resemble sober, possibly induced by covert vocalization; LORAC instead of LAROC ( $=5 \mathrm{Ss})$, Vowel transposition.

An empirical tabulation of the locus within paralog of all errors was compiled. Data were included from both the memorization trials and the retention test but errors made repeatedly by the same $S$ were counted only once. The numbers of Ss making each type of error, tabulated by locus, are shown in Table 1.

There appears to be an increasing error gradient from the first to the last portions of the paralogs. Errors at the initial letter locus are relatively infrequent. This suggests a directory-style approach operative in either
Locus Within Paralog

$\begin{array}{lrrrr}\underline{\mathrm{C}}_{1} & \underline{\mathrm{V}}_{1} & \underline{\mathrm{C}}_{2} & \underline{\mathrm{V}}_{2} & \underline{\mathrm{C}}_{3} \\ 9 & 41 & 42 & 61 & 60 \\ & 18 & 2 & 18 & \\ & 18 & & 18 & 2 \\ 4 & & 13 & & 11 \\ 1 & 6 & 3 & 6 & 2 \\ 4 & 4 & & 22 & 22 \\ & & 16 & 16 & 16 \\ & 6 & 7 & 3 & 2 \\ 18 & 93 & 83 & 144 & 115\end{array}$

memory encoding or memory search, or both. Out of 322 paralogs represented in Table 1 , the first letter was in error only 18 times, final letter errors being about 6 times as frequent. Suffix errors were about 9 times as numerous as prefix errors.

Secondly, there appears to be greater lability in the retention of vowels than in consonants, particularly in transposition errors which were 9 times as frequent for vowels as for consonants. The use of pronunciation techniques in memorizing might partly account for this since vowels tend to be more nearly interchangeable in sound than do consonants chosen at random. Also, consonants may have been studied more carefully by Ss since it is wll knwn tht cnsnnts gv wrds thr stretr wth vwls crryng lss $f$ th nfrmtn.

\section{Discussion}

The strongly limited tendencies of Ss to notice paralogs of the reversed-word and letter-change types are likely to have been due in part to the mixed-item nature of the list. Real words were perceptually salient but the special paralogs intermixed with the randomly generated ones were less apparent. Either special type, if intermixed with real words alone, might be more readily perceived and specially encoded.

Errors were induced with sufficient frequency to permit some statistical tabulation as well as examination of specific recurrent responses. Relatively common errors such as WAGIN and SOBER were partly a function of the list mixture which invited these forms as plausible responses. The within-item serial position effect in error locus is strong evidence for a directorystyle approach to memorizing. These observations provide reference points for predictions when list mixture is varied in additional research.

\section{References}

JENSEN, A. R. Spelling errors and the serial-position effect. J. educ. Psychol., 1962, 53, 105-109.

ROZOV, A. I. Irregularities in remembering: Communication II. Mistakes in remembering. Dokl. Akad. Pedag. Nauk RSFSR, 1959, No. 6, 71-73. (Only abstract seen.)

UNDERWOOD, B. J., \& HUGHES, R. H. Gradients of generalized verbal responses. Amer. J. Psychol., 1950, 63, 422-430.

Note

1. This research was performed during the junior author's tenure as an NSF Undergraduate Research Participant. 